\title{
The radial pulsation of Al Aurigae
}

\author{
L. L. Kiss ${ }^{1}$ and B. A. Skiff ${ }^{2}$ \\ 1 Department of Experimental Physics and Astronomical Observatory, University of Szeged, Dóm tér 9., 6720 \\ Szeged, Hungary \\ 2 Lowell Observatory, 1400 West Mars Hill Road, Flagstaff, AZ 86001, USA
}

Received 14 November 2000 / Accepted 12 February 2001

\begin{abstract}
We present an analysis of eleven years of Strömgren by photometry of the red semiregular variable star AI Aurigae. An early period determination of 63.9 days is confirmed by the long-term light curve behaviour. The light curve shows semi-regular changes with a mean period of 65 days reaching an amplitude of 0 m. 6 in some cycles. The $b-y$ colour changes perfectly parallel the $V$ light curve, suggesting radial oscillation to be the main reason for the observed variations. We estimate the main characteristics of the star (mass, radius, effective temperature) that suggest radial pulsation in fundamental or first overtone mode.
\end{abstract}

Key words. stars: pulsation - stars: AGB - stars: individual: AI Aur

\section{Introduction}

The light variation of semiregular variables (SR) of subtype SRa and SRb is generally associated with the pulsation of these low and intermediate mass red giants located on the asymptotic giant branch (AGB) of the Hertzsprung-Russell diagram. Since the characteristic timescale of the variations is between 20 and 2000 days (usually hundreds of days), semiregulars are typical targets of amateur observers estimating visual brightnesses. There are very few high-precision light, colour, and/or radial-velocity measurement covering many cycles that confirm the pulsational origin of the variations. Very recently, Lebzelter and his co-workers (Lebzelter 1999a,b; Lebzelter et al. 1999) presented high-precision photometric and infrared radial-velocity data for a sample of bright SR stars. Their results both illustrate the ability of automatic telescopes to monitor this type of variable stars and support the assumption of pulsation (see also Percy et al. 1996 on involving amateur photoelectric observers into regular observations of small-amplitude red variables, SARVs).

However, one cannot ad hoc accept pulsation to be the main reason of variability. As has been noted by Percy \& Polano (1998), virtually every $M$ giant is variable in brightness. This has been also supported by the largescale photometric surveys (Hipparcos, MACHO, OGLE) leading to the discovery of thousands of M-type variables. It has been pointed out by, e.g. Lebzelter et al. (2000) and Kiss et al. (2000), that specifying the physical reason responsible for the variation requires simultaneous light, colour, and radial-velocity data, as even consecutive

Send offprint requests to: L. L. Kiss,

e-mail: 1.kiss@physx.u-szeged.hu cycles may show significant deviations. This kind of longterm observation is crucial when studying the possible mechanisms affecting the light changes of SR variables. Besides pulsation, other contributors to variability cannot be readily excluded, such as time dependent surface inhomogeneities due to large convective cells (Schwarzschild 1975; Lebzelter et al. 2000), ellipsoidal deformation due to the presence of a close companion, or spots on a relatively rapidly-rotating star. Most recently, Koen \& Laney (2000) gave a detailed list of considerations concerning the possible reasons of variability and noted, that there is still no definite conclusion on this issue. Until the application of these mechanisms is better understood, each case must be studied separately.

AI Aurigae (HD 259342, 9. $9^{\mathrm{m}} 1<V<9.8, P=$ 63.9 , spectral type M5III - Chuadze 1973) is a poorlystudied red semiregular (subtype SRa) variable star. In the General Catalogue of Variable Stars (GCVS - Kholopov et al. 1985-1988) a period of $63^{\mathrm{d}} 9$ is listed with an early epoch of maximum (JD 2426029, due to Beyer 1937). Since then there has been no other photometric measurement published for this star. Due to the relatively small range of light changes, it is also neglected by the international organizations of amateur variable star observers (American Assocation of Variable Star Observers AAVSO, Association Française des Observateurs d'Étoiles Variables - AFOEV and Variable Star Observers' League in Japan - VSOLJ). The AAVSO International Database does not contain this star, AFOEV has 16 points which are wrongly identified with AI Aur (they are 2 mag brighter), and VSOLJ collected only 30 individual estimates between 1986 and 1999. Similar neglect is present concerning other observations: there was only one radial velocity monitoring 
$\left(\left\langle v_{\mathrm{r}}\right\rangle=+63 \mathrm{~km} \mathrm{~s}^{-1}\right.$, Feast et al. 1972), while Dickinson \& Dinger (1982) listed AI Aur among the negative detections during their $\mathrm{H}_{2} \mathrm{O}$ survey. There is no metallicity determination in the literature. Its infrared colours (see later) place the star among the "blue" SRs (Kerschbaum \& Hron 1992) and the presently available data suggest the star to be a regular member of this group.

The main aim of this paper is to present a continuous photometric monitoring of AI Aur between 1985 and 1996 which reveals the associated colour variations in addition to obtaining an accurate light curve. (We note, that the presented observations were acquired during a largescale photometric survey of solar type stars, Lockwood et al. 1997, without any specific reason. Therefore, this study is a by-product of that long-term observing programme). The observed behaviour of AI Aur is very likely to be mainly due to radial pulsation, most probably in fundamental or low-degree overtone mode. The next section deals with the data aquisition, while the light- and colourcurves are discussed in Sect. 3 together with the physical parameters of the star. A summary of the conclusions is given in Sect. 4.

\section{Observations}

AI Aurigae was observed on 199 nights between 12 Oct. 1985 and 8 Dec. 1996. The observations were carried out at Lowell Observatory with the $0.53 \mathrm{~m}$ telescope. The detector was a single-channel photoelectric photometer (EMI $6256 \mathrm{~S}$ tube) equipped with Strömgren $b$ and $y$ filters. The measurements were obtained almost always through a $29^{\prime \prime}$ diaphragm; some measurements in bad seeing were made through a $49^{\prime \prime}$ aperture, and in bright Moonlight a $19^{\prime \prime}$ aperture was occasionally used.

The differential magnitudes were aquired relative to HD $46159\left(V=8^{\mathrm{m}} 054, b-y=0 \cdot 623\right.$, G8III), while the photometric stability was checked against HD 259662 ( $V=9^{\mathrm{m}} 556, b-y=0^{\mathrm{m}} 639$, K0III). The data were corrected only for differential extinction using long-term monthly means for $k_{b}$ and $k_{y}$ (Lockwood \& Thompson 1986). The standard transformations are represented with slight colour terms (roughly $V=y+0.05(b-y)_{i}, b-y=$ $\left.1.08(b-y)_{i}\right)$. The internal consistency is of order of 0.005 as suggested by the rms errors of the means for the comparison stars in $y$ and $b\left(0 \cdot 0046\right.$ and $\left.0^{\mathrm{m}} \cdot 0048\right)$. The quoted standard values for the comparison stars were determined on four nights from standard star observations (extending to several very red Landolt stars). Due to the red colour of the variable, there might be some remaining systematic shift of $0 \cdot 020-0 \cdot 025$ in the $V$ brightness. In any case, the scatter of the observations is essentially negligible compared to the range of the variations. The data (mean values of var-comp and var-check) are presented in Table $1^{1}$ and plotted in Fig. 1.

\footnotetext{
${ }^{1}$ Table 1 is also available in electronic form at the CDS via anonymous ftp to cdsarc.u-strasbg.fr (130.79.128.5) or via

http://cdsweb.u-strasbg.fr/cgi-bin/qcat?J/A+A/370/496
}
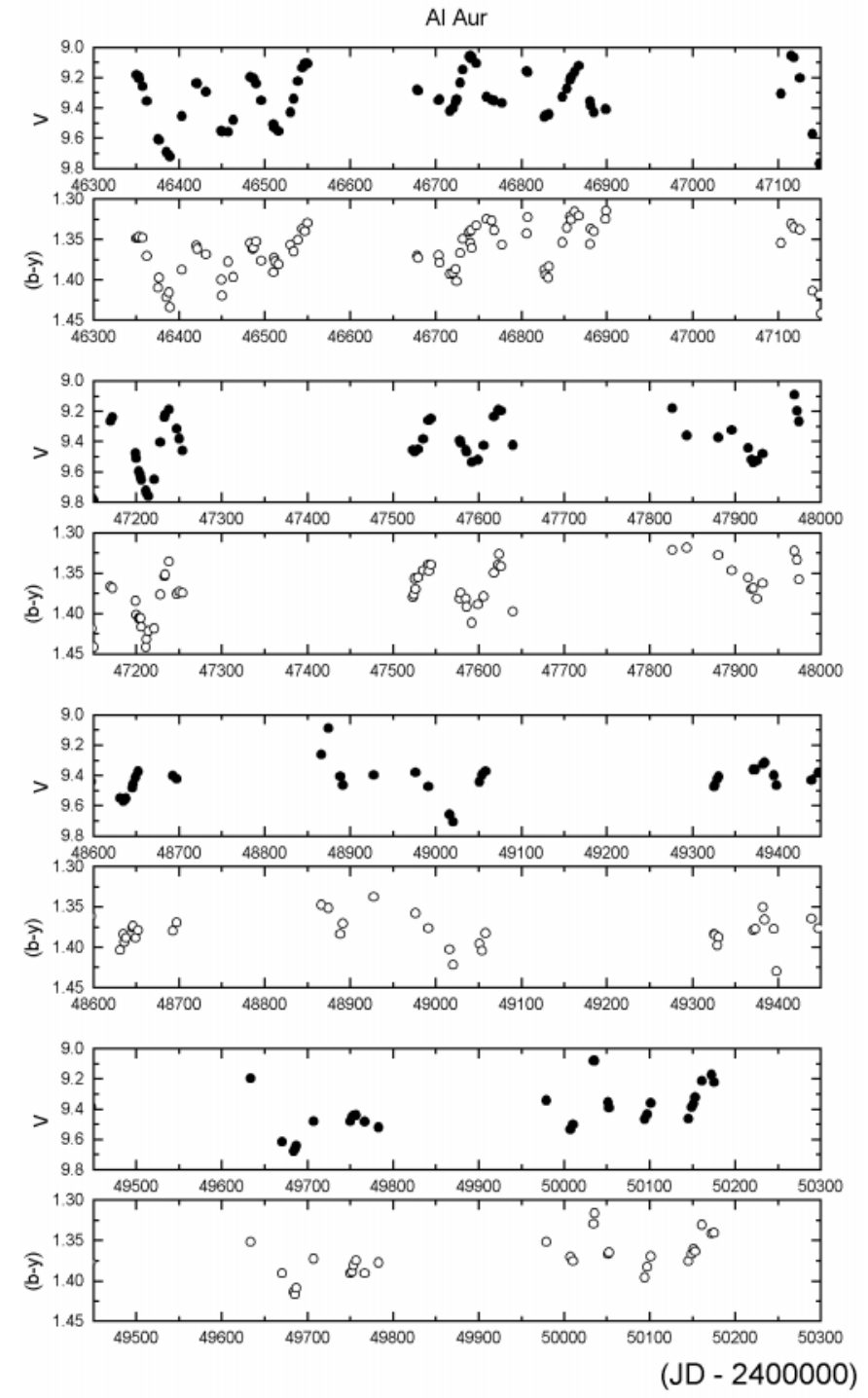

Fig. 1. The light and colour curves of AI Aur between 1985 and 1996. Note the 600-day break between MJD 48000 and 48600

\section{Discussion}

In the following, we characterize the observed light variations, discuss the importance of the colour measurements and compare AI Aur with other semiregulars with available similar data series.

\subsection{Period analysis}

Data presented in Table 1 are plotted in Fig. 1. Note that some parts are excluded from the presentation due to the sparse light curve coverage (between MJD 4800048600). However, when analysing the whole dataset, we have also used those data that are not presented in Fig. 1. Qualitatively the light curve is that of a typical semiregular without strictly repeating cycles. This has been investigated in detail by means of standard Fourier analysis implemented with Period98 of Sperl (1998). 
Table 1. Strömgren photometry of AI Aurigae (MJD = JD-2400000)

\begin{tabular}{|c|c|c|c|c|c|c|c|c|c|c|c|}
\hline MJD & $V$ & $b-y$ & MJD & $V$ & $b-y$ & MJD & $V$ & $b-y$ & MJD & $V$ & $b-y$ \\
\hline 46351.000 & 9.182 & 1.348 & 46765.900 & 9.346 & 1.330 & 47529.783 & 9.451 & 1.357 & 48991.754 & 9.477 & 1.376 \\
\hline 46352.996 & 9.192 & 1.353 & 46768.933 & 9.354 & 1.336 & 47535.796 & 9.384 & 1.348 & 49016.708 & 9.659 & 1.402 \\
\hline 46353.996 & 9.205 & 1.348 & 46777.838 & 9.367 & 1.360 & 47541.771 & 9.261 & 1.347 & 49020.617 & 9.708 & 1.427 \\
\hline 46357.987 & 9.259 & 1.352 & 46806.787 & 9.159 & 1.341 & 47542.725 & 9.261 & 1.350 & 49051.654 & 9.435 & 1.394 \\
\hline 46362.975 & 9.357 & 1.368 & 46807.771 & 9.168 & 1.329 & 47544.725 & 9.248 & 1.345 & 49054.658 & 9.396 & 1.406 \\
\hline 46375.958 & 9.609 & 1.413 & 46827.758 & 9.463 & 1.389 & 47578.683 & 9.389 & 1.382 & 49058.675 & 9.373 & 1.385 \\
\hline 46376.954 & 9.615 & 1.401 & 46828.763 & 9.450 & 1.398 & 47579.679 & 9.407 & 1.379 & 49325.792 & 9.474 & 1.382 \\
\hline 46385.942 & 9.693 & 1.421 & 46831.833 & 9.447 & 1.396 & 47585.704 & 9.455 & 1.389 & 49326.829 & 9.463 & 1.389 \\
\hline 46388.917 & 9.714 & 1.422 & 46832.750 & 9.442 & 1.385 & 47586.658 & 9.471 & 1.392 & 49329.821 & 9.425 & 1.403 \\
\hline 46389.921 & 9.717 & 1.440 & 46848.721 & 9.332 & 1.352 & 47592.658 & 9.536 & 1.414 & 49330.871 & 9.407 & 1.389 \\
\hline 46403.917 & 9.457 & 1.387 & 46853.692 & 9.275 & 1.338 & 47599.671 & 9.522 & 1.391 & 49371.754 & 9.363 & 1.378 \\
\hline 46420.813 & 9.236 & 1.361 & 46857.658 & 9.217 & 1.319 & 47606.671 & 9.423 & 1.385 & 49373.775 & 9.358 & 1.385 \\
\hline 46421.817 & 9.240 & 1.359 & 46858.683 & 9.198 & 1.325 & 47618.633 & 9.232 & 1.356 & 49382.812 & 9.326 & 1.354 \\
\hline 46431.875 & 9.297 & 1.373 & 46862.700 & 9.164 & 1.314 & 47623.633 & 9.195 & 1.342 & 49384.687 & 9.311 & 1.371 \\
\hline 46449.850 & 9.547 & 1.399 & 46867.650 & 9.127 & 1.321 & 47624.625 & 9.196 & 1.334 & 49395.692 & 9.398 & 1.382 \\
\hline 46450.742 & 9.561 & 1.420 & 46880.638 & 9.358 & 1.359 & 47626.625 & 9.194 & 1.347 & 49398.725 & 9.467 & 1.436 \\
\hline 46457.783 & 9.563 & 1.373 & 46881.658 & 9.387 & 1.336 & 47640.638 & 9.430 & 1.392 & 49439.617 & 9.435 & 1.362 \\
\hline 46463.767 & 9.483 & 1.396 & 46885.675 & 9.435 & 1.340 & 47826.929 & 9.181 & 1.321 & 49447.642 & 9.378 & 1.383 \\
\hline 46483.675 & 9.200 & 1.356 & 46898.629 & 9.408 & 1.333 & 47843.900 & 9.357 & 1.324 & 49633.958 & 9.200 & 1.349 \\
\hline 46484.662 & 9.535 & 1.380 & 46899.629 & 9.419 & 1.310 & 47880.813 & 9.375 & 1.328 & 49670.883 & 9.617 & 1.388 \\
\hline 46486.700 & 9.206 & 1.364 & 47103.987 & 9.305 & 1.358 & 47896.683 & 9.328 & 1.343 & 49684.858 & 9.678 & 1.411 \\
\hline 46487.679 & 9.205 & 1.359 & 47115.937 & 9.055 & 1.334 & 47915.692 & 9.445 & 1.353 & 49685.842 & 9.662 & 1.418 \\
\hline 46490.700 & 9.239 & 1.357 & 47118.950 & 9.066 & 1.341 & 47919.754 & 9.519 & 1.373 & 49687.800 & 9.639 & 1.411 \\
\hline 46496.667 & 9.355 & 1.373 & 47125.892 & 9.205 & 1.336 & 47921.779 & 9.539 & 1.369 & 49707.787 & 9.479 & 1.381 \\
\hline 46510.671 & 9.509 & 1.391 & 47140.846 & 9.572 & 1.416 & 47925.683 & 9.530 & 1.379 & 49750.696 & 9.480 & 1.390 \\
\hline 46511.671 & 9.529 & 1.373 & 47148.838 & 9.765 & 1.425 & 47932.683 & 9.481 & 1.366 & 49752.713 & 9.450 & 1.386 \\
\hline 46512.662 & 9.535 & 1.380 & 47150.871 & 9.786 & 1.444 & 47969.667 & 9.090 & 1.323 & 49754.692 & 9.449 & 1.378 \\
\hline 46516.650 & 9.557 & 1.382 & 47170.763 & 9.267 & 1.369 & 47972.650 & 9.195 & 1.336 & 49757.708 & 9.438 & 1.381 \\
\hline 46530.650 & 9.430 & 1.360 & 47172.829 & 9.239 & 1.365 & 47974.671 & 9.266 & 1.366 & 49767.625 & 9.484 & 1.393 \\
\hline 46534.638 & 9.345 & 1.362 & 47199.692 & 9.480 & 1.385 & 48218.854 & 9.249 & 1.374 & 49783.625 & 9.520 & 1.379 \\
\hline 46539.629 & 9.220 & 1.363 & 47200.696 & 9.511 & 1.405 & 48294.700 & 9.495 & 1.396 & 49979.942 & 9.342 & 1.358 \\
\hline 46544.650 & 9.133 & 1.335 & 47203.721 & 9.598 & 1.407 & 48296.646 & 9.501 & 1.394 & 50007.917 & 9.530 & 1.380 \\
\hline 46547.654 & 9.108 & 1.341 & 47204.729 & 9.616 & 1.410 & 48322.608 & 9.677 & 1.400 & 50010.954 & 9.502 & 1.378 \\
\hline 46550.638 & 9.113 & 1.325 & 47205.729 & 9.636 & 1.407 & 48597.858 & 9.440 & 1.368 & 50034.871 & 9.082 & 1.330 \\
\hline 46678.979 & 9.280 & 1.370 & 47206.679 & 9.651 & 1.419 & 48631.787 & 9.555 & 1.405 & 50035.875 & 9.082 & 1.319 \\
\hline 46679.979 & 9.287 & 1.375 & 47211.692 & 9.725 & 1.441 & 48635.787 & 9.566 & 1.392 & 50051.867 & 9.359 & 1.364 \\
\hline 46703.992 & 9.351 & 1.373 & 47212.692 & 9.740 & 1.431 & 48636.708 & 9.562 & 1.394 & 50052.846 & 9.389 & 1.365 \\
\hline 46704.979 & 9.351 & 1.377 & 47214.704 & 9.758 & 1.427 & 48638.812 & 9.555 & 1.391 & 50094.737 & 9.463 & 1.399 \\
\hline 46716.963 & 9.421 & 1.392 & 47221.658 & 9.654 & 1.415 & 48645.787 & 9.478 & 1.381 & 50097.696 & 9.435 & 1.384 \\
\hline 46720.954 & 9.403 & 1.389 & 47228.646 & 9.406 & 1.381 & 48646.779 & 9.459 & 1.375 & 50101.742 & 9.360 & 1.371 \\
\hline 46723.963 & 9.364 & 1.386 & 47233.667 & 9.244 & 1.357 & 48649.767 & 9.411 & 1.389 & 50145.633 & 9.460 & 1.380 \\
\hline 46724.950 & 9.343 & 1.402 & 47234.654 & 9.226 & 1.352 & 48652.754 & 9.375 & 1.378 & 50149.608 & 9.388 & 1.367 \\
\hline 46728.958 & 9.238 & 1.367 & 47238.642 & 9.191 & 1.333 & 48693.642 & 9.398 & 1.389 & 50151.608 & 9.363 & 1.364 \\
\hline 46731.950 & 9.149 & 1.354 & 47247.654 & 9.317 & 1.382 & 48697.633 & 9.420 & 1.373 & 50153.608 & 9.319 & 1.368 \\
\hline 46739.921 & 9.061 & 1.337 & 47250.658 & 9.380 & 1.375 & 48866.975 & 9.259 & 1.358 & 50161.617 & 9.211 & 1.337 \\
\hline 46740.971 & 9.057 & 1.357 & 47254.646 & 9.461 & 1.375 & 48874.967 & 9.088 & 1.355 & 50172.650 & 9.175 & 1.344 \\
\hline 46741.946 & 9.074 & 1.338 & 47523.808 & 9.455 & 1.383 & 48888.925 & 9.408 & 1.382 & 50175.621 & 9.224 & 1.342 \\
\hline 46742.954 & 9.062 & 1.360 & 47524.838 & 9.457 & 1.377 & 48891.904 & 9.466 & 1.366 & 50366.967 & 9.338 & 1.362 \\
\hline 46747.933 & 9.104 & 1.335 & 47525.787 & 9.467 & 1.363 & 48927.879 & 9.396 & 1.340 & 50425.900 & 9.386 & 1.357 \\
\hline 46759.933 & 9.326 & 1.328 & 47526.783 & 9.459 & 1.373 & 48976.812 & 9.384 & 1.355 & & & \\
\hline
\end{tabular}

First, we have calculated the power spectrum of the whole dataset. It is shown in Fig. 2, where the insert shows the window function. One main peak is present at $f=0.01604 \mathrm{c} / \mathrm{d}(A=0 \mathrm{~m} 14)$ corresponding to a period of 62 d 345 . After a simple prewhitening with this frequency, the power spectrum of the residuals contains a closely separated frequency $(f=0.01548 \mathrm{c} / \mathrm{d}, A=0.10)$. However, it is a direct consequence of the unstable period, since the whole dataset could be fitted neither with a single period nor with a sum of two close periods. Therefore we studied the light curve in four separate subsets (each being about 1000 days long) 


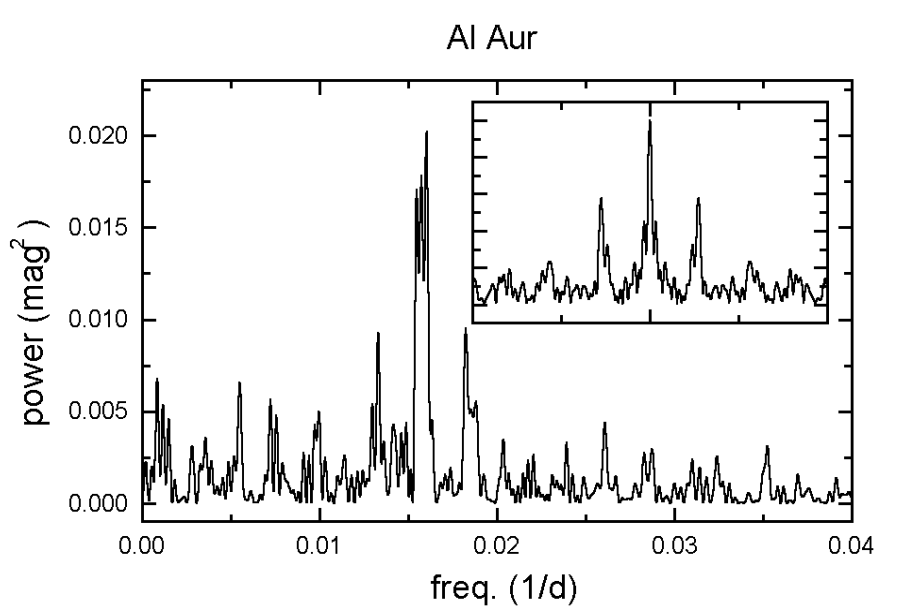

Fig. 2. The Fourier spectrum of the whole dataset. The insert shows the window function with the same frequency scale

plotted in the left panels of Fig. 3. We performed similar frequency analysis as for the whole light curve and the results can be summarized as follows:

1. There is a well-defined main peak in every Fourier spectra representing the average cycle length varying around the mean period of $\sim 65$ days. The fitted curves (solid lines in Fig. 3) have the following periods and amplitudes: $1-63.2,0.2 ; 2-67^{\mathrm{d}} .1,0.13 ; 3-100 \mathrm{~d}$, 0.12 (the few data points may result in wrong result); 4-65 d, 0. 15 . The typical period uncertainty is about $1 \mathrm{~d}$ (except for the third subset);

2. The first subset can be fitted very well with two harmonic components, however the second one is simply due to the variation of the mean brightness. Later data do not support the presence of this false period ( $\sim 860$ days $)$;

3. The phase diagrams of the individual subsets (right panels of Fig. 3) suggest the average light curve shape to be quite stable. The data were phased with those periods listed above;

4. The prewhitened spectra of the subsets do not contain any other significant peak higher than $0{ }^{\mathrm{m}} 08$. We have not tried to use the low-amplitude component(s), because the residual light curves show irregular rather than cyclic behaviour. We feel that when analysing light curves of SR variables the noise level in the frequency spectrum is only partly due to the observational errors and it would be misleading to accept every peaks in the spectrum being much higher than the mean error of the observations (about 0 . 01 in our case). The star itself may grossly increase the noise of the light curve through its erratic behaviour caused, e.g., by the mild chaos affecting the pulsation (see e.g. Buchler \& Kolláth 2000). It is well established, e.g. for Mira stars, that radial pulsation alone may produce irregularities in the light and/or radial velocity curves (for instance, through shock wave propagation in the extended atmosphere, Bertschinger \& Chevalier 1985; Fox \& Wood 1985). The complicated stellar responses on other physical mechanisms (coupling between pulsation and convection, possible departures from the spherical symmetry) prevent drawing a simple and straightforward conclusion on the irregularities. Most recently, Lebzelter et al. (2000) revived the old idea proposed first by Schwarzschild (1975) on the large convective cells introducing some irregularity in the light variation. However, in the case of AI Aur, only speculations can be drawn;

5. The results slightly depend on the selection of the subsets (we have tried different lengths starting from one year to the finally accepted 1000 days); on the other hand, they are strongly affected by the lack of points in the second half of the data.

Considering these points we conclude that the light curve cannot be described with simple sums of harmonic functions, but it has a slowly and apparently irregularly variable characteristic cycle length (instead of period) near 65 days. The period analysis of the $b$-filter data leads to the same conclusion.

\subsection{Colour variations}

The conclusions presented so far are typical for semiregular variables (see, e.g. Kiss et al. 1999). The novelty in our results is the presence of colour measurements. To our knowledge, there have not been really long-term (covering tens of cycles) time-series multicolour data in the literature for any semiregular variable star. Although Hipparcos Epoch Photometry (ESA 1997) contains typically 100-200 points for thousands of variables, the limited time span does not permit accurate period determination for red variables. High-precision $V$-band observations of different SRs were presented, for instance, by Percy et al. (1989), Cristian et al. (1995), Percy et al. (1996) and Lebzelter (1999), but none of these studies dealt with the colour variations.

One of the most extensive time-resolved multicolour photometric surveys was published by Smak (1964), who reported $U B V$ observations of twenty-nine Mira stars and twelve semiregulars during two years, covering typically only one cycle per star. He did not find any clear correlation of the $B-V$ colour and the $V$ brightness for the SR stars. Jerzykiewicz (1984) studied the light and colour variations of HD 157010 (V818 Her), concluding that the star's $U B V$ magnitudes are effective temperature parameters through the sensitivity of the continuous spectrum and TiO blanketing. Wisse (1981) presented $U B V$ observations for $35 \mathrm{SRs}$ for classification purposes. He found that well-defined correlations and anti-correlations exist among the variables, where the phase difference of the $V$ and $B-V$ maxima is either 0 or 0.5. Cadmus et al. (1991), in their study of three SRs, noted only that the colour changes were small compared to the changes in brightness. Narrow-band observations of SR variables in the literature are quite scanty. An interesting application can be found in Wasatonic \& Guinan (1998), who used three colors of 
Al Aurigae
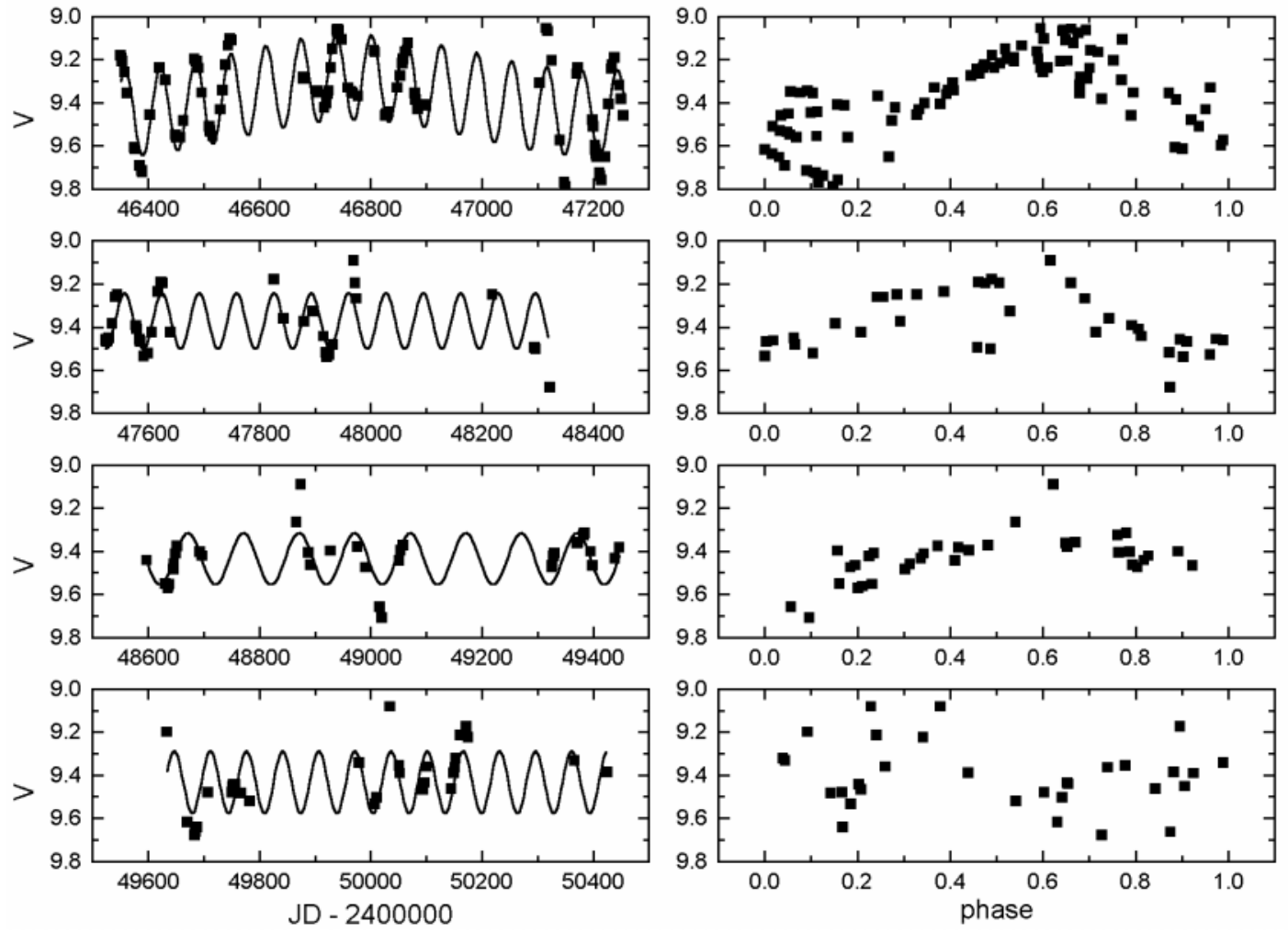

Fig. 3. The four subsets and the corresponding phase diagrams of AI Aur

the Wing near-infrared system to trace the temperature and radius variations of $\mathrm{V} \mathrm{CVn}$. These authors measured the highest temperature (from an infrared index) around the maximum light.

The most important feature of our observations is the almost perfectly parallel light and colour curves. This is a characteristic behaviour in radially pulsating variables, where the temperature maximum occurs close to the maximum light. It means that the star appears bluer when it is at maximum light, which is in good agreement what is expected from pure thermal variations driven by pulsation. In the case of FS Comae (SR star with $P \approx 58 \mathrm{~d}$ ), Torres et al. (1993) reached the opposite conclusion based on $U B V$ data series. The star showed reversed colour changes explained by the fact that $B-V$ colour in FS Com is a molecular absorption index, rather than a temperature indicator. The Strömgren $b$ filter (centered near $4700 \AA$ ) has a relatively clear spectral region in the center of the passband, but the wings on both sides have moderately-weak TiO absorption, particularly the 2,0 transition with bandhead at $4761 \AA$. The $y$ filter is not so strongly affected, with only the usual $\mathrm{Fe}$, Ti, etc. lines. Unlike the $B-V$ colour, $b-y$ is completely unaffected by the wide TiO bandhead at $\sim 5167 \AA$. We conclude that the observations imply radial oscillation with dominant thermal effects as the main reason for the light variability. The $\sim 0^{\mathrm{m}} 1$ amplitude of the $b-y$ curve corresponds to a temperature change of $<300 \mathrm{~K}$ (as estimated from the synthetic colour grids of Kurucz 1993).

\subsection{Stellar parameters}

Adopting radial pulsation for AI Aur, further constraints can be drawn on the basic stellar parameters. Unfortunately, there is no parallax measurement for AI Aur, thus only spectroscopic parallax or various empirical period-colour-luminosity relations (e.g. Barthès et al. 1999) can be used to determine its luminosity. We have chosen the former approach, because we wanted to estimate stellar properties without any assumption on its pulsation. In the following we have neglected the interstellar reddening since the observed mean $b-y$ colour (1.35) is close to the expected value for an M5 giant star (see Jorissen et al. 1995 for Strömgren photometry of red giants).

The IRAS [12]-[25] colour is $-1^{\mathrm{m}} 41$. Following the definitions by Kerschbaum \& Hron (1992), this means that AI Aur belongs to the "blue" semiregulars (no indication for circumstellar shells, $P<150^{\mathrm{d}}, T_{\text {eff }}>3200 \mathrm{~K}$ ). The infrared brightnesses $\left(K=2^{\mathrm{m}} \cdot 71, I=6^{\mathrm{m}} 18\right)$, taken from the IRC catalogue (Neugebauer \& Leighton 1969), combined with the mean $V$ magnitude imply colour indices $V-K=6 \cdot{ }^{\mathrm{m}} 8$ and $V-I=3 \cdot{ }^{\mathrm{m}} 3$. The latter value results in a $T_{\text {eff }}=3420 \mathrm{~K}$ using the temperature scale by Dumm \& Schild (1998). A less biased estimate can be inferred from twenty M5III-type stars in the sample of Dumm \& Schild (1998): $\left\langle T_{\text {eff }}(\mathrm{M} 5 \mathrm{III})\right\rangle=3520 \pm 100 \mathrm{~K}$. For an independent check we have used the $(V-K)-(710-888)$ and $(I-K)-(710-888)$ relations in Alvarez et al. (2000) 
resulting in $T_{\text {eff }}=3300 \mathrm{~K}$ from their $T_{\text {eff }}-(710-888)$ calibration. Similarly, one can get a mean radius and mass for the given spectral type of $\langle R\rangle=123 \pm 34 R_{\odot}$ and $\langle M\rangle=2.0 \pm 0.8 M_{\odot}$. Adopting these spectral type-mass and spectral type-radius values we calculated a pulsational constant $Q\left(=P \cdot \sqrt{\rho / \rho_{\odot}}\right)=0.065_{-0.03}^{+0.06}$. Recently, Percy \& Parkes (1998) discussed the pulsation modes in smallamplitude red variable stars reaching a conclusion that some stars are likely to pulsate in up to the third overtone mode. Within these frameworks AI Aur seems to pulsate in the fundamental or first overtone mode as is suggested by theoretical models of Xiong et al. (1998) or Ostlie \& Cox (1986). The overtone pulsation is favoured by the position of AI Aur in the $K$-band P-L diagram of long-period variables derived by Bedding \& Zijlstra (1998) from Hipparcos parallaxes. For this, we estimated $M_{K}$ from the $V-K$ colour and $M_{V}$-spectral-type calibration of Thé et al. (1990). The resulting $M_{K}=-7{ }^{\mathrm{m}} 5$ and $\log P \approx 1.8$ place AI Aur close to the upper sequence in Fig. 1 of Bedding \& Zijlstra (1998), which may be interpreted as a consequence of pulsation in a different mode than that valid for most Mira stars (represented by the lower sequence in Fig. 1 of Bedding \& Zijlstra 1998).

Finally, there is an interesting period-gravity relation for a wide range of radially pulsating variable stars presented by Fernie (1995), which can also be used to test the assumption of radial oscillation. The adopted mass and radius give a $\log g=0.56 \pm 0.4$ which is coincidentally the same as predicted by Eq. (1) of Fernie (1995). Plotting AI Aur in Fig. 1 of Fernie (1995), its position is as deviant as that of Mira itself suggesting the first overtone to be somewhat more likely. However, the universality of this period-gravity relation has no firm theoretical background and verification; therefore, this comparison should be considered only as a possible hint for the mode of pulsation.

The presented considerations are on the whole consistent with the recent observational and theoretical results regarding the mode of pulsation in Mira and semiregular variables. For example, Feast (1996) found that semiregular variables, independently of their metallicity, pulsate probably in the first overtone mode. Further supporting arguments were listed by Feast (1999). However, semiregulars form a quite heterogeneous group, in which stars may pulsate in the fundamental, or 1st, 2nd or even 3rd overtone mode, as has been clearly demonstrated by Wood et al. (1999). That is why every individual case study has to be performed without any definite preconception.

\section{Conclusions}

The main results presented in this paper can be summarized as follows:

1. New Strömgren by photometric observations of the semiregular variable AI Aurigae are presented and discussed. The period analysis of the whole light curve confirms the early period determination of 63.9 days.
However, the light curve shows typical semiregular behaviour with changing amplitude and cycle length;

2 . The $b-y$ colour measurements revealed highly paralel light and colour variations. Since the $b-y$ colour is considerably good temperature indicator (rather than molecule indicator, as is the $B-V$ colour), the phase dependent light and temperature changes resemble classical radially pulsating stars. The observations imply radial oscillation with dominant thermal effects as the main reason for light variability;

3. We estimated the basic stellar parameters, such as the effective temperature $(3520 \pm 100 \mathrm{~K})$, mean radius $\left(123 \pm 34 R_{\odot}\right)$, mass $\left(2.0 \pm 0.8 M_{\odot}\right)$. The spectral type-mass and spectral type-radius values give a pulsational constant of $0.065_{-0.03}^{+0.06}$. The results are consistent with radial pulsation in the fundamental or (more likely) first overtone mode. The period-gravity relation of Fernie (1995) also supports this conclusion.

Acknowledgements. This research was supported by the "Bolyai János" Research Scholarship of LLK from the Hungarian Academy of Sciences, Hungarian OTKA Grant \#T032258 and Szeged Observatory Foundation. The NASA ADS Abstract Service was used to access data and references. This research has made use of the SIMBAD database, operated at CDS-Strasbourg, France.

\section{References}

Alvarez, R., Lançon, A., Plez, B., \& Wood, P. R. 2000, A\&A, 353,322

Barthès, D., Luri, X., Alvarez, R., \& Mennessier, M. O. 1999, A\&AS, 140, 55

Bedding, T. R., \& Zijlstra, A. A. 1998, ApJ, 506, L47

Bertschinger, E., \& Chevalier, R. A. 1985, ApJ, 299, 167

Beyer, M. 1937, AN, 262, 257

Buchler, J. R., \& Kolláth, Z. 2000, in Nonlinear Stellar Pulsation, ed. M. Takeuti, \& D. Sasselov (Astrophysics and Space Science Library, AASL), in press

Cadmus, R. R. Jr., Willson, L. A., Sneden, C., \& Mattei, J. A. 1991, AJ, 101, 1043

Chuadze, A. D. 1973, Byull. Abastumanskaya Astrofiz. Obs., 44, 105

Cristian, V. C., Donahue, R. A., Soon, W. H., et al. 1995, PASP, 107, 411

Dickinson, D. F., \& Dinger, A. S. C. 1982, ApJ, 254, 136

Dumm, T., \& Schild, H. 1998, New Ast., 3, 137

ESA 1997, The Hipparcos and Tycho Catalogues, ESA SP-1200

Feast, M. W. 1996, MNRAS, 278, 11

Feast, M. W. 1999, IAU Symp., 191, 109

Feast, M. W., Woolley, R., \& Yilmaz, N. 1972, MNRAS, 158, 23

Fernie, J. D. 1995, AJ, 110, 2361

Fox, M. W., \& Wood, P. R. 1985, ApJ, 297, 455

Jerzykiewicz, M. 1984, AcA, 34, 353

Jorissen, A., Mowlavi, N., Sterken, C., \& Manfroid, J. 1995, A\&A, 324, 578

Kholopov, P. N., Samus, N. N., Frolov, M. S., et al. 1985-88, General Catalogue of Variable Stars, $4^{\text {th }}$ edition (Nauka, Publishing House, Moscow) (GCVS4)

Kerschbaum, F., \& Hron, J. 1992, A\&A, 263, 97 
Kiss, L. L., Szatmáry, K., Cadmus, Jr. R. R., \& Mattei, J. A. 1999, A\&A, 346, 542

Kiss, L. L., Szatmáry, K., Szabó, Gy., \& Mattei, J. A. 2000, A\&AS, 145, 283

Koen, C., \& Laney, D. 2000, MNRAS, 311, 636

Kurucz, R. L. 1993, ATLAS9 Stellar Atmosphere Programs and $2 \mathrm{~km} \mathrm{~s}^{-1}$ Model Grids, CD-ROM, No. 13

Lebzelter, T. 1999a, A\&A, 346, 537

Lebzelter, T. 1999b, A\&A, 351, 644

Lebzelter, T., Hinkle, K. H., \& Hron, J. 1999, A\&A, 341, 224

Lebzelter, T., Kiss, L. L., \& Hinkle, K. H. 2000, A\&A, 361, 167

Lockwood, G. W., \& Thompson, D. T. 1986, AJ, 92, 976

Lockwood, G. W., Skiff, B. A., \& Radick, R. R. 1997, ApJ, 485,789

Neugebauer, G., \& Leighton, R. B. 1969, Two micron sky survey: A preliminary Catalogue, Calif. Inst. Technology, NASA
Ostlie, D. A., \& Cox, A. N. 1986, ApJ, 311, 864

Percy, J. R., \& Parkes, M. 1998, PASP, 110, 1431

Percy, J. R., Landis, H. J., \& Milton, R. E. 1989, PASP, 101, 893

Percy, J. R., Desjardnis, A., Yu, L., \& Landis, H. J. 1996, PASP, 108, 139

Smak, J. 1964, ApJS, 9, 141

Sperl, M. 1998, Comm. Astr. Seis., 111

Thé, P. S., Thomas, D., Christensen, C. G., \& Westerlund, B. E. 1990, PASP, 102, 565

Torres, G., Mazeh, T., Latham, D. W., \& Stefanik, R. P. 1993, PASP, 105, 360

Wasatonic, R., \& Guinan, E. F. 1998, IBVS, No. 4579

Wisse, P. N. J. 1981, A\&AS, 44, 273

Wood, P. R., Alcock, C., Allsman, R. A., et al. (The MACHO Collaboration) 1999, IAU Symp., 191, 151

Xiong, D. R., Deng, L., \& Cheng, Q. L. 1998, ApJ, 499, 355 